\title{
Trauma-focused group psychotherapy is not effective for posttraumatic stress disorder in Vietnam veterans
}

Schnurr PP,Friedman MJ, Foy DW et al. Randomized trial of trauma-focused group therapy for posttraumatic stress disorder. Results from a Department of Veterans Affairs cooperative study. Arch Gen Psychiatry 2003;60:481-489.

\section{QUESTION: Is trauma-focused group therapy more effective than present-centred therapy, which avoids discussion of the trauma, for posttraumatic stress disorder in Vietnam veterans.}

Design

Randomised controlled trial.

\section{Setting}

10 Veterans Affairs medical centres, USA.

\section{Participants}

360 male Vietnam veterans with posttraumatic stress disorder not receiving other psychological treatments (apart from the 12-step programme for substance abuse). Almost all had a previous history of mood disorder or substance abuse. Veterans with other significant psychiatric disorders, severe cardiovascular disorders or cognitive impairment were excluded.

\section{Intervention}

30 weeks of trauma-focused group psychotherapy, delivered weekly in groups comprising 6 people, plus 5 booster sessions at monthly intervals, versus control intervention of present-centred comparison treatment. In the trauma focused therapy group, each participant received 2 sessions dedicated to their own trauma; and had to listen to an audiotape of these sessions at least 8 times. The intervention additionally comprised psychoeducation, cognitive restructuring, relapse prevention and coping skills training. Present-centred therapy avoided trauma-focused references and other components of trauma-focused therapy. Both intervention and control group sessions included education about posttraumatic stress disorder and coping skills.

\section{Main outcome measures}

Severity of posttraumatic stress disorder symptoms measured using the Clinician Administered PTSD Scale (CAPS), and PTSD checklist.

\section{Main results}

Sources of funding: Grant CSP 420 from the VA Cooperative Studies Program.

\section{Correspondence to:} Dr P Schnurr, VA

National Center for PTSD (Mail Stop

116D), VA Medical and Regional Office Center, White River Junction, VT 05009 email:

paula.schnurr@ dartmouth.edu
Intention to treat analysis found no significant difference between treatment groups in severity of posttraumatic stress disorder symptoms at 12 months. However, symptoms improved from baseline in both treatment groups (CAPS score 80.4 at baseline and 72.8 at 12 months with trauma-focused group therapy; CAPS score 82.0 at baseline and 74.8 at 12 months with present-centred therapy; PTSD checklist score 61.8 at baseline and 58.9 at 12 months with trauma-centred therapy; PTSD checklist score 62.6 at baseline and 60.0 at 12 months with present-centred therapy; $\mathrm{p}<0.05$ for all within group comparisons). There was a higher drop out rate in the trauma-focused group (23\% v 9\%, $\mathrm{p}<0.001)$

\section{Conclusions}

Trauma-focused group therapy is no more effective than present-centred group therapy for post-traumatic stress disorder in Vietnam veterans.

\section{COMMENTARY}

There is now good evidence that both psychological and pharmacological treatments can effectively reduce post traumatic stress disorder (PTSD) symptoms. ${ }^{1}$ Individual exposure therapy is widely accepted as one of the most effective treatments for PTSD but there has been limited research into the efficacy of group therapy. ${ }^{2}$

This study is one of the largest and most ambitious of all PTSD treatment trials to date. The authors' desire to evaluate trauma-focused group therapy (TFGT) under conditions that translate readily to clinical practice resulted in a design that would be considered flawed by RCT purists. Aspects that make the results difficult to interpret include the fact that over two thirds of participants had other psychiatric disorders co-morbid with their PTSD, that medication could be changed during the study, the significant differences in outcome between different sites, that Vietnam veterans attending the VA system are notoriously difficult to treat and that the only control condition was another active group therapy.

It appeared that TFGT modestly reduced symptoms of PTSD but the magnitude of change was very small (mean symptom reduction of less than $10 \%$ ) and no different to that of the control group. Importantly over one third of those in the TFGT dropped out, significantly more than dropped out of the control group. It is unlikely that TFGT will become widely used as a result of this study. However, given the issues described above it is probably premature to completely discard TFGT and it would be interesting to see if an adaptation was more effective in non-veteran PTSD sufferers. With the benefit of hindsight I wonder if the researchers wished they had conducted a more tightly designed efficacy trial in the first instance than a study that appears to have raised more questions than it has answered.

Jonathan I Bisson, MD MRCPsych Consultant Liaison Psychiatrist and Honorary Senior Lecturer Cardiff E Vale NHS Trust Cardiff, UK

1 Bisson J. Post-traumatic stress disorder. Clinical Evidence 002: 7:913-9

2 Foa E, Keane T, Friedman M, eds. Effective treatments for PTSD: practice guidelines from the intern tudies. New York, NY: Guilford Publications; 2000. 